\title{
Paraplegia
}

\section{Spinal Injury Rehabilitation: Do Staff and Patients Agree on What They are Talking About?}

\author{
C. A. Glass, BSc, MClin Psych, PhD, CPsychol, K. R. Krishnan, MB, FRCS, \\ J. D. Bingley, RGN \\ Mersey Regional Spinal Injuries Centre, Promenade Hospital, Leicester Street, \\ Southport, PR9 OHY, UK.
}

\section{Summary}

The psychological effects of spinal cord injury on patients themselves have been discussed in a number of articles, but few studies have been made of patients and staff, perceptions of the effectiveness of the treatment they receive and supply.

An earlier investigation by the present authors showed that there were differences between the views of staff and of patients in terms of their understanding of rehabilitation. As a consequence, a number of procedural changes were implemented in the Spinal Unit, in an attempt to improve the information provided for patients and their relatives, and the selection and support of staff.

Reanalysis of the staff and patients views of rehabilitation were carried out 12 months later using a standardised questionnaire and any changes in response were noted.

The present findings are analysed, and the potential implications for other units are noted.

Key words: Spinal injury; Rehabilitation; Psychological aspects; Treatment.

Throughout the spinal rehabilitation literature, there is a paucity of objective examination of the perceptions of either patients, or staff themselves, of the efficacy of the rehabilitation process.

Tucker (1980) emphasised that the emotional adjustment of both patients and staff was essential for successful rehabilitation. It is considered that such perceptions are of great importance to achieve optimal rehabilitation, especially during the in-patient phase, as clarity of rehabilitation procedures enables specific and discernible goals to be set, and levels of individual rehabilitation attainment to be monitored.

Correspondence and reprint requests to: Mrs S. Cribb, Administrator, Mersey Regional Spinal Injuries Centre, Promenade Hospital, Leicester Street, Southport, PR9 0HY, UK.

This paper was originally presented at the 28th Annual Scientific Meeting of the International Medical Society of Paraplegia, Rome, 1989. 
A paper published by the present authors (Krishnan et al., 1988), examined how job satisfaction amongst nurses affected the turnover rate in the Regional Spinal Injuries Centre in Southport, UK. This was achieved by using a standardised measure of staff and patient estimation of the ward environment (Moos, 1974), which contained 10 discrete sub-scales; measuring individual staff and patients' perceptions for the degree of involvement, support, spontaneity, autonomy, practical orientation, personal problem orientation, anger and aggression, order and organisation, programme clarity, and staff control in the Unit.

Members of staff were divided into those who had less than 1 year experience of spinal injury patient nursing, and those who had more than a years experience. Patients were similarly divided, dependent on whether they were first-time new admissions, or if they had been admitted for post-trauma care or surgery.

One-way factorial analysis of the four groups data showed that there were significant differences in the responses to four of the ten subscales of the Moos' Scale. Tukey a posteriori contrast coefficients were calculated for each of these four subscales and showed that staff of less than a years experience had greater levels of autonomy and involvement than did the other three groups; whilst first admission patients scored significantly lower than did the other three groups on practical orientation and programme clarity.

Two conclusions were drawn from the study:

1. New admission patients do not receive sufficient information to allow them to actively engage in the rehabilitation process.

This was not seen simply as a problem during the initial phases of intensive care, where a degree of uncertainty might be expected because of the variability in rehabilitation rates, but extended throughout the rehabilitation process. Patients, for example, at 5 and 6 months into their rehabilitation programme were sometimes unclear about which goals they still had to achieve.

2. Inexperienced staff displayed unrealistically high expectations of the efficacy of the rehabilitation programme for spinally injured patients.

This might in part explain the high staff turnover. Realistic target setting has been suggested to be a major factor in job satisfaction (Locke et al., 1981). Thus under conditions where expectations of effectiveness are unrealistically high, it might be expected that greater experience would lead to disillusionment and lower moral. The staff member may of course try to accommodate the cognitive dissonance between expected and actual success by modifying his/her expectation, which would explain the differences between the experienced and inexperienced staff found in our study.

However two findings make such a conclusion open to question:

1. Correlations of length of service with the scores obtained on the individual subscales were non-significant, suggesting that such a change is unlikely or, at least does not occur in any 'stepwise' modification towards more realistic goals.

2. The high staff turnover itself suggests that the accommodation of dissonance 
between expectation and actual outcome is not a major factor. Indeed, the high turnover rate implies that a more likely explanation of the differences in the perceptions of the experienced and inexperienced staff are that those with unrealistically high expectations leave the Unit.

Interpretations of the reasons for staff leaving a Spinal Unit are not necessarily related to their expectations for patient rehabilitation. Staff stay and leave for many different reasons. The recently reported regrading of nurses in the United Kingdom because of annual wastage into fields with better prospects shows that this is not merely a problem for Spinal Units.

Similarly lack of understanding about the rehabilitation goals for individual patients may relate to the lack of available information and communication between patients and staff, or may relate to an unwillingness or inability to assimilate such information due to a lack of psychological 'preparedness'; the use of denial as an adaptive function has been shown in other areas of trauma (Glass, 1988), and staff will be aware of the difficulties in encouraging patients to understand instructions during the initial period of shock immediately posttrauma.

\section{Method}

\section{Intervention}

Following on from this initial study a number of changes in the practice of the Unit were implemented.

Staff interview protocol. In an attempt to select staff whose role expectancies more closely match the rehabilitative demands of the patient, the protocol for staff appointments has been modified to provide detailed information on the demands of the individual role, the range of patient problems to be encountered, and an explanation of the importance of psychological factors in promoting active rehabilitation.

In-service staff training. As an adjunct to the above, a member of the nursing staff has responsibilities for in-service training and draws upon the expertise of all the other disciplines involved in the rehabilitation process in order to produce a constantly updated package of new procedures and modes of interaction. The involvement of staff in support groups, and the development of patient forums, to discuss matters of individual and common concern adds to the general atmosphere of communication and cooperation.

Patient information. With the previously measured lack of programme clarity in mind, the Centre has produced a handbook* for patients, which contains information about the physical effects of trauma, the likely complications, an explanation of the aims of rehabilitation, and an explanation of the unit philosophy and personnel. This book is designed to be given to the patient soon after the individual prognosis has been explained by the Consultant, Care Coordinator, and Clinical Psychologist, and is used both for individual study, and as a basis for discussion during regular rehabilitation sessions.

${ }^{*}$ Copy of handbook available from the Centre. 
Relatives' information. The lack of active involvement of relatives in rehabilitation from the day of admission, is possibly one of the commonest failings of most Centres across the United Kingdom. A primary goal when a new patient is admitted is to involve the relatives, who are themselves in shock, and to provide support for the injured person. In so doing the involvement in practical activity and displacement activities also tends to reduce their anxieties, and reduces the pressure on staff at this time. In order to facilitate this process, relatives are also given a small handbook* which outlines the practical aspects associated with the first few weeks of admission; how to get to the Centre, important phone numbers, who the various staff are, what the injury means, when the person will be told their prognosis, and where to stay locally.

Having gone some way to improve not only the situation for staff, but also provide information for the patients and their relatives, it was considered appropriate to reassess the ward environment using the standardised measure developed by Moo's (1974) used in the initial investigation.

\section{Re-appraisal}

Twelve months after the introduction of the procedural changes, staff and patients were again asked to complete the Ward Atmosphere Scale (Moos; 1974). Staff were divided on the basis of time since appointment, and patients on the basis of whether they were new admissions or re-admissions. Four groups of respondents were therefore obtained:

a. Staff with less than 1 year spinal injury experience

b. Staff with over 1 year experience of spinal injury

c. Patients who had been admitted with a spinal injury

d. Patients who had received a spinal injury in the past and were now rehospitalised for secondary conditions.

All patients and nursing staff at the Centre were asked to complete the questionnaire. Tetraplegics were assisted, where necessary, by non-staff visitors.

Analysis of the data obtained were conducted on the basis of total staff and patient results, as in the original Moos studies, although given the nature of the responses noted in the earlier investigation (Krishnan et al., 1988) analyses were also carried out on the four categories of respondent outlined above.

\section{Results}

Questionnaires were given out to 22 nursing staff and 26 patients.** Return rates for the two groups were $20(91 \%)$ and $22(85 \%)$ respectively. Of the 20 staff returns 13 were from staff with more than 1 year of spinal injury experience, with 11 of the 22 patient returns received from first admission patients.

No significant differences could be found between the means from the overall patient means and the overall staff means when compared with the respective British norms provided by Moos (1974).

*Copy of handbook available from the Centre.

**The Unit contains 35 beds, but patients with non-traumatic lesion were not included in the investigation. 
Table Group mean and standard deviations (in parentheses) for each of the ten sub-scales of the Ward Atmosphere Scale (Moos, 1974). Staff and patient norms from the original Moos article are included in the latter two columns for purposes of comparison

\begin{tabular}{lcccccc}
\hline \multirow{2}{*}{ Subscale } & \multicolumn{3}{c}{ Group } & means & \multicolumn{3}{c}{ Norms. for } \\
& $\mathrm{A}$ & $\mathrm{B}$ & $\mathrm{C}$ & $\mathrm{D}$ & $\mathrm{A}+\mathrm{B}$ & $\mathrm{C}+\mathrm{D}$ \\
\hline Involvement & $5 \cdot 6$ & $3 \cdot 9$ & $5 \cdot 1$ & $4 \cdot 4$ & $5 \cdot 5$ & $4 \cdot 3$ \\
& $(2 \cdot 0)$ & $(1 \cdot 7)$ & $(2 \cdot 9)$ & $(1 \cdot 9)$ & $(1 \cdot 7)$ & $(1 \cdot 3)$ \\
Support & $6 \cdot 4$ & $7 \cdot 2$ & $5 \cdot 7$ & $5 \cdot 4$ & $7 \cdot 0$ & $6 \cdot 0$ \\
& $(1 \cdot 8)$ & $(1 \cdot 8)$ & $(3 \cdot 4)$ & $(2 \cdot 5)$ & $(1 \cdot 1)$ & $(1 \cdot 2)$ \\
Spontaneity & $5 \cdot 7$ & $5 \cdot 9$ & $5 \cdot 2$ & $4 \cdot 2$ & $5 \cdot 8$ & $4 \cdot 8$ \\
& $(2 \cdot 8)$ & $(1 \cdot 0)$ & $(1 \cdot 5)$ & $(1 \cdot 8)$ & $(1 \cdot 1)$ & $(0 \cdot 9)$ \\
Autonomy & $4 \cdot 3$ & $3 \cdot 5$ & $3 \cdot 9$ & $4 \cdot 7$ & $5 \cdot 5$ & $4 \cdot 8$ \\
& $(1 \cdot 1)$ & $(0 \cdot 8)$ & $(2 \cdot 0)$ & $(1 \cdot 3)$ & $(1 \cdot 1)$ & $(0 \cdot 9)$ \\
Practical orientation & $7 \cdot 0$ & $7 \cdot 5$ & $6 \cdot 2$ & $5 \cdot 8$ & $6 \cdot 7$ & $5 \cdot 5$ \\
& $(1 \cdot 9)$ & $(1 \cdot 3)$ & $(2 \cdot 6)$ & $(1 \cdot 8)$ & $(1 \cdot 2)$ & $(1 \cdot 0)$ \\
Personal problem orientation & $3 \cdot 3$ & $3 \cdot 0$ & $4 \cdot 3$ & $1 \cdot 9$ & $5 \cdot 3$ & $4 \cdot 2$ \\
& $(2 \cdot 6)$ & $(2 \cdot 1)$ & $(2 \cdot 8)$ & $(1 \cdot 4)$ & $(1 \cdot 1)$ & $(1 \cdot 0)$ \\
Anger and aggression & $6 \cdot 9$ & $6 \cdot 2$ & $5 \cdot 2$ & $4 \cdot 8$ & $5 \cdot 4$ & $4 \cdot 3$ \\
& $(1 \cdot 2)$ & $(1 \cdot 6)$ & $(2 \cdot 1)$ & $(1 \cdot 0)$ & $(1 \cdot 6)$ & $(1 \cdot 3)$ \\
Order and organisation & $5 \cdot 6$ & $5 \cdot 3$ & $6 \cdot 1$ & $4 \cdot 1$ & $6 \cdot 6$ & $6 \cdot 4$ \\
& $(1 \cdot 6)$ & $(1 \cdot 0)$ & $(3 \cdot 2)$ & $(1 \cdot 5)$ & $(1 \cdot 8)$ & $(1 \cdot 7)$ \\
Programme clarity & $6 \cdot 0$ & $5 \cdot 3$ & $3 \cdot 8$ & $2 \cdot 6$ & $7 \cdot 2$ & $5 \cdot 4$ \\
Staff control & $(2 \cdot 0)$ & $(1 \cdot 4)$ & $(2 \cdot 8)$ & $(1 \cdot 6)$ & $(0 \cdot 9)$ & $(1 \cdot 1)$ \\
& $0 \cdot 9$ & $1 \cdot 4$ & $3 \cdot 1$ & $2 \cdot 8$ & $2 \cdot 4$ & $4 \cdot 0$ \\
Number in each group & $(0 \cdot 9)$ & $(1 \cdot 0)$ & $(1 \cdot 2)$ & $(1 \cdot 2)$ & $(0 \cdot 9)$ & $(1 \cdot 3)$ \\
& 7 & 13 & 11 & 11 & &
\end{tabular}

Key: $A=$ Staff with less than 1 year experience.

$\mathrm{B}=$ Staff with greater than 1 year experience.

$\mathrm{C}=$ First admission patients.

$\mathrm{D}=$ Readmission patients.

One way factorial analyses of variance were performed on the responses on each subscale by each of the four staff and patient sub-groups. The mean scores for each are shown in the Table together with the normative data provided by Moos (1974). Significant differences between the groups were found on two of the sub-scales: programme clarity- $(\mathrm{F} 3, \mathrm{df} / \mathrm{error}=5.57, \mathrm{p}>\cdot 05)$ and staff control- $(\mathrm{F} 3$, $\mathrm{df} /$ error $=9 \cdot 25, \mathrm{p}>\cdot 05)$.

Tukey a posteriori contrast coefficients were calculated for both of these subscales and indicated that the variance in programme clarity was due to the low scores attained by those patients who were readmissions (Group D), whilst the variance in staff control was attributable to the lower scores provided by those staff of less than one year experience (Group A).

\section{Discussion}

It is interesting to note that the significant differences in staff and patient groupings responses to questions examining their perceptions of programme clarity are maintained, despite the implementation of procedures specifically designed to reduce this. Programme clarity refers to the extent to which the patient knows what to expect in the day-to-day routine of the ward. However, closer examination of the data indicates that the lack of clarity is recorded by readmission patients; the group who during the earlier investigation reported programme clarity to be 
significantly higher than the other three groups, now providing results which are significantly lower.

The likely explanation of this alteration in perception is due to the effects of the interventions specifically implemented to increase new admissions, and their relatives, awareness of what to expect during the first few months of rehabilitation. It is possible that the results reflect increased awareness in this group rather than decreased understanding by the readmission patients.

The reason for the significant difference in the responses of the four groups to the staff control sub-scale is less clear. Staff control measures the extent to which it is necessary for staff to restrict patients; for example, in the strictness of rules and schedules, in the relationships between patient and staff, and in measures taken to keep patients under effective control.

That staff with less than 1 year spinal experience score lower on this sub-scale than the other three groups may simply be a reflection of their own uncertainty about the amount of control which they feel is necessary. In those situations where there is a degree of uncertainty, such as whether a patient is allowed out for an evening, staff will usually refer to the nurse in charge. It is interesting to note that the two staff groups score lower than the two patient groups, perhaps indicating that whilst staff report that the degree of control is low, patients see the procedures in operation as restrictive.

If the results of the present investigation are compared with those prior to the implementation of the changes in information provision and training procedures (Krishnan et al., 1988) the overall lack of difference between the four sub-groups of staff and patients can be seen in a positive light, implying that the four interventions (with staff, patients and relatives) have achieved some success.

It is unfortunate that the individual effects of each specific intervention could not be assessed, though the demands upon service provision made this impractical. It therefore remains possible to conclude that the changes in staff and patients perceptions of the Spinal Unit vary simply as a function of time, and in fact have little to do with the changes in practice which have been implemented. However, a number of factors make the drawing of such a conclusion inappropriate.

The current staff turnover, though reduced to some extent, meant that the staff who completed the questionnaire as 'new' staff on this occasion of testing were entirely different to those who completed the questionnaire in the earlier investigation. The lack of statistical significance between their scores and the current, more experienced, staff would indicate an increase in commonality of responding.

Similarly, the lack of variability in the two patient groups' scores would indicate a change in awareness which, given that the 'new' patient groups in both investigations were independent, is unlikely to be a function of time. The mean scores for the new and re-admission patients (though not directly comparable) across both occasions of testing do show some interesting trends.

This is particularly true for the current scores on programme clarity, as both patient groups score lower than on first testing. It is unlikely that this relates to a lack of understanding on the part of patients of the availability of rehabilitation options, given the increase in provision of information and dialogue. What is more probable is that the scores reflect patients' frustration at not being able to be involved in such activities at the time of the investigation (e.g. due to pressure sores 
or physical inability to engage in that activity during the early stages of rehabilitation).

The primary motivation for conducting the initial appraisal, and this current reappraisal, of the perceptions of staff and patients of the rehabilative efficacy of the Spinal Centre, was to assess why so many staff were leaving. It was noted in the earlier investigation that interpretations of staff reasons for leaving are not entirely related to their expectations for patient progress. Whilst it is not possible to analyse the data for significance the reduction in nursing turnover from 12 to 7 in the Southport Spinal Centre is seen as a positive outcome.

It must be remembered that staff and patients interact continually and the effects of positive and negative communications from either source will influence all subsequent interactions. By objectively examining the views of both staff and patients' perceptions of rehabilitation it becomes possible to produce interactive care plans (whereby the patient and staff from all disciplines are involved in working towards the goals of rehabilitation together) which are essentially contractual agreements.

In summary, therefore, the present investigation has attempted to show that the use of an objective measure of rehabilitation has practical value, not only in terms of assessing the prevailing rehabilitation atmosphere, but also in enabling the effects of specific interventions to be monitored.

It is considered that the use of the current and other such measures has implications for the assessment of the quality of service provided in all spinal centres, allowing for continued monitoring and reappraisal of service provision, and could be utilised in a similar way in many other medium to long stay placements.

\section{References}

GLASS CA 1988 An investigation of some psychological factors of importance in the assessment and treatment of sexual functioning in male patients undergoing haemodialysis and with kidney transplants. Unpublished $\mathrm{PhD}$ thesis, University of Liverpool.

KRISHNAN KR, GLASS CA, JACKSON HF, BINGLEY JD 1988 Patient and nursing staff perceptions of living and working on a spinal injuries unit. Paraplegia 26:287-292.

LOCKE EA, SHAW KN, SARRI LM, LATHAM GP 1981 Goal setting and task performance 1969-1980. Psychological Bulletin 90: 125-152.

Moos R 1974 The Ward Atmosphere Scale. Consulting Psychologists Press Inc., California, USA.

TUCKER SJ 1980 The psychology of spinal cord injury: patient-staff interaction. Rehabilitation Literature 41(5-6): 114-121:160. 